\title{
Coherent dual-band 2x4 MIMO radar experiment exploiting photonics
}

\author{
Salvatore Maresca ${ }^{(1)}$, Leonardo Lembo ${ }^{(1,2)}$, Filippo $\operatorname{Scotti}^{(3)}$, Giovanni Serafino ${ }^{(1)}$, Antonio Malacarne ${ }^{(1)}$, Paolo Ghelfi2 ${ }^{(3)}$, \\ Antonella Bogoni ${ }^{(1,3)}$ \\ (1) Scuola Superiore Sant'Anna, Via Moruzzi 1, 56124 Pisa, Italy, Email: antonella.bogoni@cnit.it \\ (2) Italian Navy, Naval Research Center CSSN, Vallauri Institute, Viale Italia 72, 57100 Livorno, Italy
}

(3) CNIT, Via Moruzzi 1, 56124 Pisa, Italy

\begin{abstract}
In this paper the first coherent dual-band 2x4 MIMO radar experiment is presented. Range/cross-range maps demonstrate the higher cross-range resolution due to the coherence and the enhanced performance introduced by dual-band operation
\end{abstract}

\section{Introduction}

After more than one century since its invention mankind needs radars more than ever for manifold applications ranging from long-established air surveillance to pollution control via satellite observations, driving assistance, biomedical purposes or even more innovative applications. No other sensing system including high resolution cameras, infrared and light detection and ranging (LIDAR) sensors, has the same efficiency and reliability as radar systems have in terms of "all day" and "all weather" operability. Modern applications require a precise and accurate localization and classification of the targets. In a conventional monostatic radar, range resolution depends on the signal bandwidth, whereas angular resolution on the antenna features. In general, angular resolution is rather modest with respect to range resolution. Therefore in the last decades diverse processing techniques have been developed to address this critical aspect, leading to complex data processing. Nevertheless, limitations still exist.

On the other hand, albeit range resolution is far better than angular resolution, there are wide margins for its improvement. However, nowadays, due to communication spectrum erosion and difficulties to efficiently manage signals with high fractional bandwidths in the radio frequency (RF) domain, the future radars could not use broader radar waveform bands to fulfill demanding resolution requirements.

In this framework the only alternative is to change the paradigm from current monostatic (i.e. stand-alone) and single-band radar systems to unprecedented networks of spatially distributed and multi-band radars. Their enormous potential stands in their capability of observing the same scene from different viewpoints, with benefits when trying to detect targets characterized by high angular radar cross section (RCS) variability (i.e. complex or stealth targets). Recently, a novel class of multistatic radar systems has been proposed, named multiple-input multiple-output (MIMO) radars. Differently form multistatic radars, i.e. multiple monostatic independent radars, MIMO radars consist of multiple radars operating in sinergy. In fact, a MIMO radar is a system employing multiple transmit waveforms and having the ability to jointly process all signals received by multiple antennas [1]. MIMO radars exploit spatially distributed information to achieve an excellent cross range (i.e. angular) resolution independent of antenna features. The coherent elaboration of data collected from different positions can contribute to further increase the radar network detection and localization capabilities. Moreover, the multi-band operation allows to synthesize larger bands to fulfill the future resolution requirements. Again, coherence among bands allows to avoid heavy synchronization algorithms for coherent data processing. The implementation of coherent multi-band MIMO radars is limited by several $\mathrm{RF}$ issues related to the generation and manipulation of frequency-agile RF signals and their coherent distribution as well.

In the last decade several microwave solutions have been proposed and demonstrated for overcoming these problems. The use of photonics has been demonstrated for radar signal generation and elaboration, providing extreme frequency flexibility, coherent multiband operation and software defined radar configuration [2][4]. Photonics has been also used for assuring coherent RF signal distribution. The benefits of the coherent distributed sensor operation have been already numerically investigated [5],[6].

Recently the authors presented preliminary results on coherent MIMO radar systems exploiting 2 transmitters and two receivers ( $2 \times 2$ configuration) [7].

In this paper, the first coherent dual-band 2x4 MIMO radar experiment is presented. The proposed system is obtained exploiting photonics both for coherent multiband RF signal generation and detection and for their distribution to the spatially distributed radar antennas.

\section{Photonics-based MIMO radar asystem}

Photonics-based RF up- and down-conversion

Conventional generation and detection of RF signals are generally realized through multiple electronic up- and down-conversion stages; each stage, is constituted by an 
electronic local oscillator and an electronic mixer followed by a band pass filter to select the up-converted signal and suppress any other undesired frequency. Unfortunately, each up-conversion stage introduces a nonnegligible phase and amplitude noise, due to the local oscillators and the nonlinearities of the active electronic mixers, increasing when the RF get higher. Moreover the tunability of these schemes is rather poor. Instead a photonics-based up/down-conversion is based on the concept of heterodyning two lasers in a photodiode, thus producing an RF signal whose frequency is equal to the detuning between the two lasers. Moreover, if one of the lasers is modulated, the heterodyning operation transfers the modulation to the carrier frequency given by the lasers detuning. The availability of tunable lasers and broadband $(>40 \mathrm{GHz})$ photodiodes and modulators allows for frequency-agile RF signal generation and detection. In the photonics-based up- and down-conversion the modulator acts as an electro-optical converter moving the radar waveform from baseband or intermediate frequency to the optical domain, while the photodiode acts as an optoelectrical converter transferring the radar waveform from the optical to the RF domain. The use of multi-frequency optical comb, also allows for the generation and detection of coherent multi-band radar signals. An optical comb can be implemented thorough a mode locked laser (MLL), assuring extremely low phase noise [8].
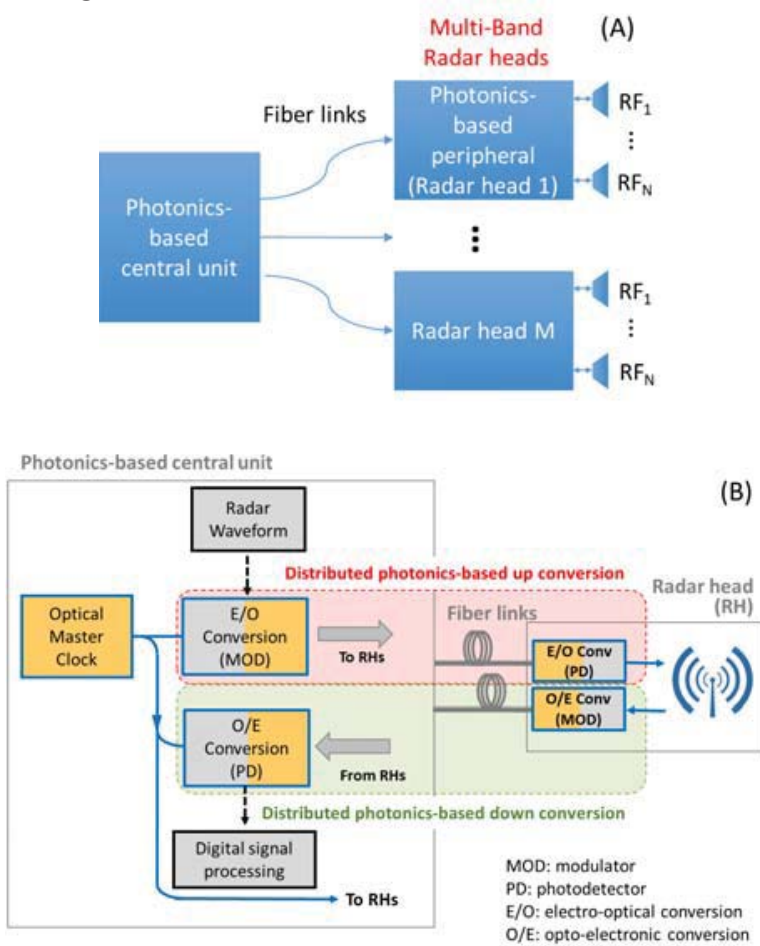

Figure 1. (A): Working principle of a photonics-based coherent MIMO radar network; (B): detailed structure of a photonicsbased distributed up- and down-conversion.

\section{Photonics-based coherent MIMO radar signal distribution}

Once the radar signals are loaded on an optical carrier (i.e. after the electro-optical convertion), optical fibers can be exploited for an efficient signal distribution, from/to the central unit to/from the MIMO radar antennas. In fact, fiber transmission is broadband, low-loss $(0.2 \mathrm{~dB} / \mathrm{km})$, and immune to electromagnetic (EM) interference. Moreover, optical fiber allows transporting the RF signal over long distances ( $>$ several $\mathrm{kms}$ ) without significant distortions, and preserving the signal coherence. Finally, optical fiber is light, small, and flexible, and therefore can be installed in complex and narrow sites, such as unmanned vehicles or satellites. After radar signal distribution, at the antenna sites, the optical signals are transferred to the RF through the opto-elctrical converter. In this way, distributed photonics-based up- and downconversions are implemented.

Therefore, with reference to Figure 1 (A), a photonicsbased MIMO radar can be composed of a photonic-based central unit and several antenna sites or radar heads (RHs). The central unit distributes and collects via optical fiber, the $\mathrm{N}$ RFs $\left(\mathrm{RF}_{1} \ldots \mathrm{RF}_{\mathrm{N}}\right)$ signals where $\mathrm{N}$ is the number of employed carrier frequencies to/from all the RHs. Figure. 1 (B) reports the schematic structure of the MIMO radar network: the distributed photonics-based upand down-conversions are implemented with a stage in the central unit, shared among all the RHs, and a further dedicated stage in each $\mathrm{RH}$. From a processing point of view the coherent MIMO elaboration corresponds to a digital beamforming (where coherence is fundamental for the cross-range resolution enhancement) using a sparse antenna array. The sparse configuration of the antenna elements (i.e. the distributed RHs) results in the presence of side lobes that seriously affects the system performance. Their presence may lead to false target detection and localization errors. However, these side lobes has been demonstrated that can be reduced acting on the geometry of the radar system and on the number of RHs.

\section{Coherent dual-band 2x4 MIMO system experiment}

At the best of our knowledge, we implemented here the first example of coherent dual-band $2 \times 4$ (i.e. 2 transmitters and 4 receivers) MIMO radar system.

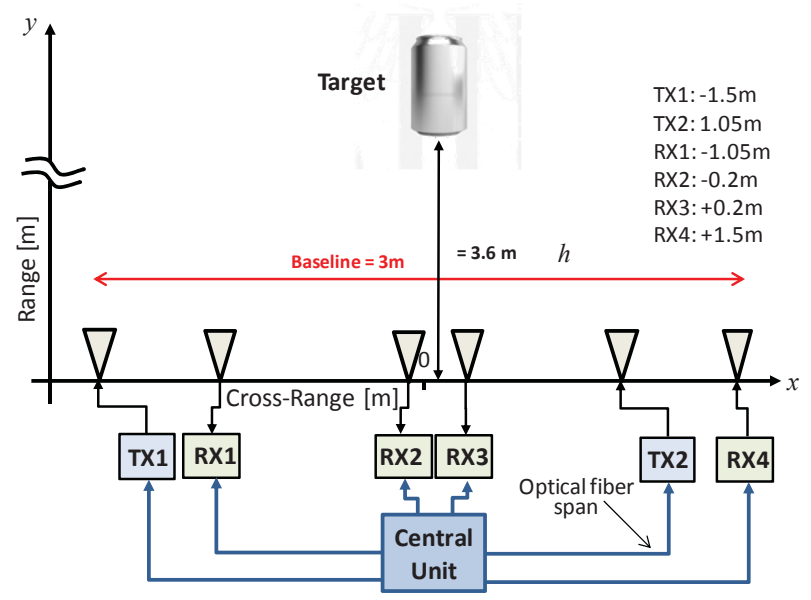

Figure 2. Experimental setup of the coherent dual-band $2 \times 4$ MIMO radar system 
Frequency-modulated continuous-wave (FM-CW) signals has been used, which consent to maximize the transmitted power and to manage ultra-wide bandwidth signals. Linear chirped signals with bandwidth of $400 \mathrm{MHz}$ are considered. TX1 and TX2 transmit an up- and down-chirp respectively. Furthermore, the aforementioned signals have been separated also in the time domain in order to limit mutual interferences and ensure an appropriate signal orthogonality. In particular, the two waveforms are interleaved so that each TX is active for one pulse repetition interval (PRI) and is silent for the following PRI, when it is the turn of the other TX to send its signal. Here a 273 ns-long PRI has been selected corresponding to a non ambiguous range of about $41 \mathrm{~m}$. largely sufficient for the present scenario. The used RFs are $8 \& 9 \mathrm{GHz}$. As reported in Fig. 2, the antennas are placed on a 1D 3mlong baseline thus enabling 2D imaging. The precise location of each antenna is indicated in the figure too. Horn antennas with $17 \mathrm{dBi}$ of gain and path antenna with $5 \mathrm{dBi}$ of gain have been used at the transmitters and receivers respectively. The lunched power is $10 \mathrm{dBm}$. The used target is a cylinder can with a radar cross section (RCS) of $-7 \mathrm{dBsm}$ (assumed omnidirectional on the rangeazimuth plane) at a distance of $3.6 \mathrm{~m}$.
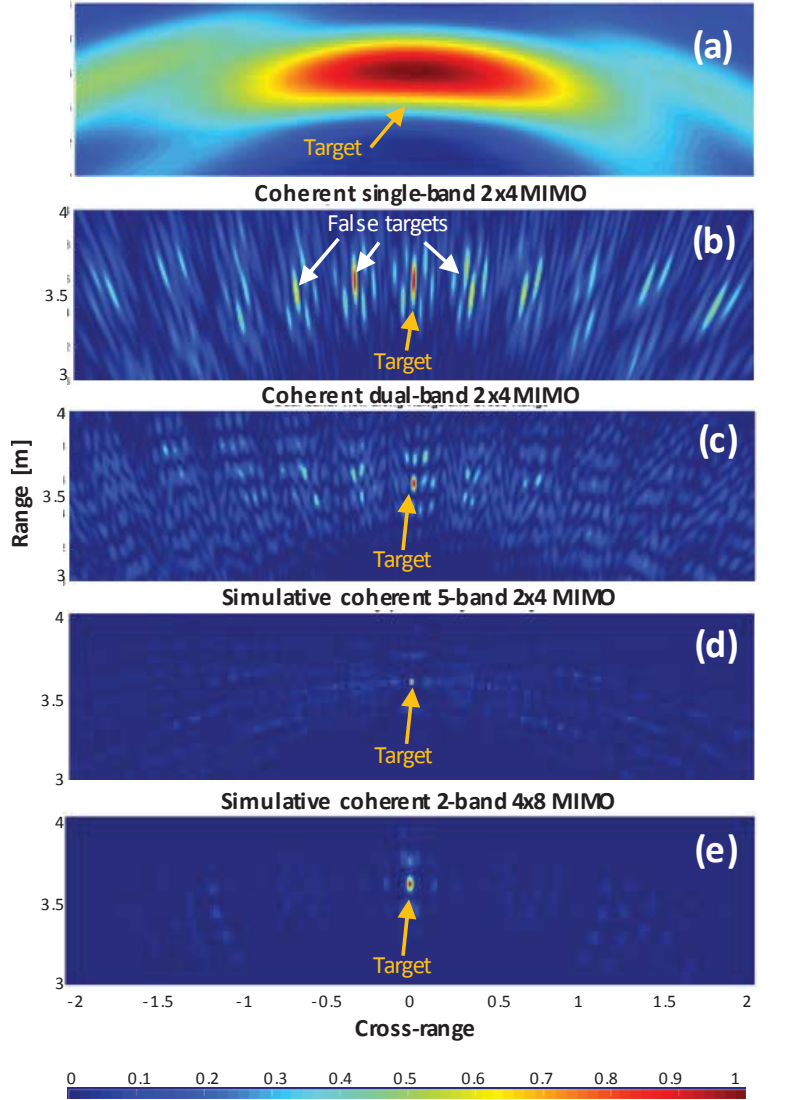

Figure 3. Experimental range/cross-range ambiguity map using a $2 \times 4$ configuration and single-band operation with non-coherent (a) or coherent (b) MIMO processing, and with dual-band operation and coherent processing (c). Simulative range/cross range map using a $2 \times 4$ configuration and 5-band operation with coherent MIMO processing (d), and using a $4 \times 8$. configuration and dual-band operation with coherent MIMO processing (e)
Non-coherent and coherent MIMO radar processing tools have been developed as reported in [7]. Figure 3 (A) reports the experimental range/cross-range ambiguity map using a $2 \times 4$ configuration and single-band operation $(8 \mathrm{GHz})$ with non-coherent MIMO processing. The crossrange resolution is larger than 1 meter, while applying a coherent MIMO processing the cross-range resolution improves down to $3 \mathrm{~cm}$. The range resolution depends on the signal bandwidth and it does not change in the two non-coherent and coherent cases. Unfortunately, due to the sparsity of the MIMO array configuration the coherent processing introduces not negligible sides lobes which may lead to false detections. A multi-band operation can help in decreasing these side lobes. Figure 3 (c) reports the experimental results exploiting a dual-band configuration. It is evident the considerable side lobe reduction. By simulation we investigated the system performance, by further increasing the number of used bands. Figure 3 (D) reports the range/cross range ambiguity map in the case of 5 bands, where the side lobes are further reduced with an extinction ratio $>10 \mathrm{~dB}$. The range resolution is also improved due to the multband operation, while a total band equal to the sum of the used bands is synthesized. The numerical analysis also confirms that an alternative solution to eliminate the side lobes consists in increasing the number of sensors. Figure 3 (E) reports the results in the case of dual band operation with $4 \times 8$ sensors.

\section{Conclusions}

The paper reported the first experiment of a coherent dual-band 2x4 MIMO experiment where photonics is exploited for guaranteeing coherence among all the radar signal and radar bandwidths. The experimental results demonstrate the importance of radar signal coherence for increasing of almost two orders of magnitude the crossrange resolution. Moreover, the dual-band operation allows for a strong reduction of the side lobes that origins by the sparse configuration of the sensors. By simulation it has been also verified the impact on the system performance of a multiband operation and of the number of the sensors.

\section{Acknowledgements}

This work has been partially supported by the NATO project SOLE

\section{References}

1. H. Godrich et all, "Target Localization Accuracy Gain in MIMO Radar-Based Systems" IEEE Transactions on Information Theory, Vol. 56, N. 6, June 2010.

2. P. Ghelfi, et al., "Photonics for Radars Operating on Multiple Coherent Bands", invited paper, J. Lightwave Technol., vol. 34, n. 2, 2016

3. P.Ghelfi et al, "A fully photonics-based coherent radar system”, Nature Vol 507 March 2014. 
4. F.Laghezza et all, "Field Evaluation of a photonicsbased radar system in a maritime environment compared to areference commercial seonsor, IET Radar Sonar \& Navigation Journal, Vol 9, n. 8, 2015.

5. G. Serafino et al., "A Photonic Approach for On-Board and Ground Radars in Automotive Applications", IET Radar Sonar \& Navigation

Journal, Vol 12, n.10, 2018.

6. L. Lembo et al, "Analysis of a Coherent Distributed MIMO Photonics-Based Radar Network", EuRAD. 2018.

7. L. Lembo, et al., "In-Field Demonstration of a Photonic Coherent MIMO Distributed Radar Network," Proc. of IEEE Radar Conference 2019, Boston (MA), USA.

8. G. Serafino, et al., "Phase and amplitude stability of EHF-band radar carriers generated from an active modelocked laser," Journal of Lightwave Technology, vol. 29, n. 23, Dec. 2011. 